\title{
Dynamical Stability Limit for the Charge Density Wave in $\mathrm{K}_{0.3} \mathrm{MoO}_{3}$
}

\author{
R. Mankowsky, ${ }^{1,2}$ B. Liu, ${ }^{1}$ S. Rajasekaran, ${ }^{1}$ H. Y. Liu, ${ }^{1}$ D. Mou, ${ }^{3}$ X. J. Zhou, ${ }^{3}$ R. Merlin, ${ }^{4}$ M. Först, ${ }^{1}$ and A. Cavalleri ${ }^{1,2,5}$ \\ ${ }^{1}$ Max Planck Institute for the Structure and Dynamics of Matter, 22761 Hamburg, Germany \\ ${ }^{2}$ University of Hamburg, 22761 Hamburg, Germany \\ ${ }^{3}$ Beijing National Laboratory for Condensed Matter Physics, Institute of Physics, \\ Chinese Academy of Sciences, Beijing 100190, China \\ ${ }^{4}$ Department of Physics, University of Michigan, Ann Arbor, Michigan 48109-1040, USA \\ ${ }^{5}$ Department of Physics, Oxford University, Clarendon Laboratory, Oxford OX1 3PU, United Kingdom
}

(Received 12 December 2016; published 15 March 2017)

\begin{abstract}
We study the response of the one-dimensional charge density wave in $\mathrm{K}_{0.3} \mathrm{MoO}_{3}$ to different types of excitation with femtosecond optical pulses. We compare direct excitation of the lattice at midinfrared frequencies with injection of quasiparticles across the low energy charge density wave gap and with charge transfer excitation in the near infrared. For all three cases, we observe a fluence threshold above which the amplitude-mode oscillation frequency is softened and the mode becomes increasingly damped. We show that all the data can be collapsed onto a universal curve in which the melting of the charge density wave occurs abruptly at a critical lattice excursion. These data highlight the existence of a universal stability limit for a charge density wave, reminiscent of the Lindemann criterion for the melting of a crystal lattice.
\end{abstract}

DOI: 10.1103/PhysRevLett.118.116402

One-dimensional charge-density waves (CDWs) are a prototypical example of a broken symmetry state, in which the energy is lowered by the modulation of the conduction electron density at a wave vector $q_{\mathrm{CDW}}$ that nests two regions of the Fermi surface [1]. This modulation in the real space charge distribution is typically also associated with a distortion of the lattice [2] and with the appearance of new excitations that can be observed in optical or Raman spectroscopy. Charge density waves are especially interesting in systems in which nesting occurs between two different bands, for which the order is often incommensurate with the lattice, resulting in highly anomalous and nonlinear dc conductivity.

Here, we study the response of the one-dimensional charge density wave material $\mathrm{K}_{0.3} \mathrm{MoO}_{3}$ (blue bronze) to short pulse optical excitation, which so far has been investigated extensively at near-infrared wavelengths [3-5]. In these studies, a collapse of the CDW gap [6] and the rearrangement of the lattice along the coordinate of the amplitude mode [7] were reported.

We seek to provide a new perspective into these dynamics by inducing melting of the same charge density wave by three different types of optical stimulation. We study excitation of the lattice alone, of charge quasiparticles immediately above the low-energy CDW gap and of charges across a high-energy charge transfer resonance. The third mechanism is the one studied in previous experiments and is analyzed here as a reference point. We find evidence for a universal dynamical instability that occurs always at specific lattice displacement, which we estimate to be of approximately $20 \%$ of the CDW-induced equilibrium lattice distortion.
$\mathrm{K}_{0.3} \mathrm{MoO}_{3}$ is a prototypical one-dimensional chargedensity wave material, made up of conducting chains of corner-sharing $\mathrm{MoO}_{6}$ octahedra along the $b$ direction of its monoclinic crystal structure [see Fig. 1(a)]. Below $T_{C}=183 \mathrm{~K}$, incommensurate CDW order with an energy gap $2 \Delta=130 \mathrm{meV}$ develops at a temperature dependent wave vector $q_{\mathrm{CDW}}=\left(1, q_{b}, \overline{0.5}\right)$, with $q_{b}=0.748$ at $100 \mathrm{~K}$ [8]. The corresponding lattice distortion is mostly transverse, involving Mo-O bond length changes perpendicular to the $b$ direction on the order of $5 \mathrm{pm}[9,10]$. The Mo-O (a)
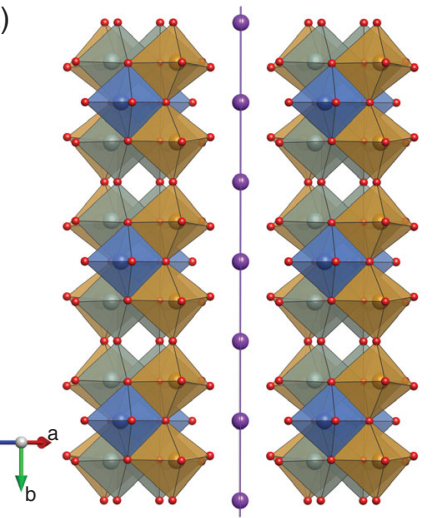

(b)

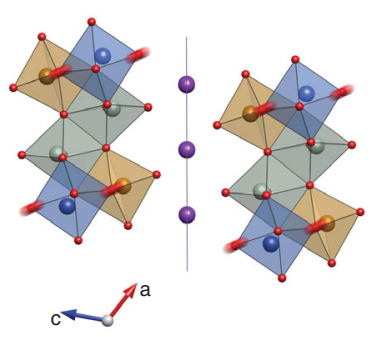

FIG. 1. (a) Monoclinic crystal structure of $\mathrm{K}_{0.3} \mathrm{MoO}_{3}$. Cornersharing $\mathrm{MoO}_{6}$ octahedra form chains along the [010] direction that are separated by sheets of potassium atoms (purple). Below $T_{C}=183 \mathrm{~K}$, incommensurate charge density wave order develops at a wave vector $q_{\mathrm{CDW}}=\left(1, q_{b}, \overline{0.5}\right)$, with $q_{b}=0.748$ at $100 \mathrm{~K}$. (b) Top view of the chains. The resonantly excited phonon mode comprises Mo-O stretching along the insulating [-201] direction, perpendicular to the chains. 
chains are separated from each other by sheets of potassium atoms making this material insulating in the perpendicular directions with an energy gap of $1.3 \mathrm{eV}$.

Because of their strong coupling to the electronic order, several phonon modes are folded back to the Brillouin zone center and appear in infrared [11] and Raman spectra $[12,13]$. The amplitudes, frequencies, and damping of these collective excitations hold important information on the density of the ordered charges [14].

Our experiments were performed on $\mathrm{K}_{0.3} \mathrm{MoO}_{3}$ single crystal, cooled to a $20 \mathrm{~K}$ base temperature, well below the transition temperature. Midinfrared pulses of 150 fs duration, with few $\mu \mathrm{J}$ of energy and tunable between 6 and $15 \mu \mathrm{m}$ wavelength, were used to resonantly excite the infrared-active $1000 \mathrm{~cm}^{-1} \mathrm{Mo}-\mathrm{O}$ stretching mode polarized along the [-201] crystallographic direction [see Figs. 1(b) and 2(a)]. Significant coupling of this phonon mode to the CDW order is known to occur at equilibrium, as evidenced by a prominent reshaping in the optical conductivity across the metal-to-CDW transition [15].

In a parallel set of experiments, the charge density wave itself was optically excited in the same wavelength range, using midinfrared pulses that were polarized along the [010] axis and tuned to the CDW gap [red area in Fig. 2(b)]. For comparison, melting of the condensate was also studied using near-infrared pulses tuned to wavelengths above the
1.3-eV charge transfer gap and polarized along [-201], perpendicular to the $\mathrm{MoO}_{6}$ octahedra chains [gray area in Fig. 2(a)].

In all three cases, the $\mathrm{K}_{0.3} \mathrm{MoO}_{3}$ reflectivity at $800 \mathrm{~nm}$ wavelength was measured as a function of time delay using 35-fs pulses, polarized along the chain direction [010]. As the penetration depths of the different pump pulses were in all cases larger than the one of the 800 -nm probe pulses, the dynamical response in the sample reflectivity can directly be compared.

Figure 2(c) shows the time-resolved reflectivity changes for the three different excitation conditions. We report here excitation at low fluence, far below the charge density wave melting threshold [6]. In all three cases, we found a prompt reduction and double-exponential recovery in the reflectivity ascribed to electronic dynamics in earlier studies $[3,4,6]$. Coherent oscillations of multiple zone-folded Raman modes were observed along with the incoherent response, dominated by the charge density wave amplitude mode at $1.68 \mathrm{THz}$. By subtracting a double-exponential fit to the incoherent electronic background, we obtained the purely oscillatory response [Fig. 3(a)], which we fitted by the sum of three exponentially decaying oscillations with frequencies of the amplitude mode at $1.68 \mathrm{THz}$ and the first doublet of zone-folded Raman modes at 2.23 and $2.56 \mathrm{THz}$ [Fig. 3(b)]. (a)

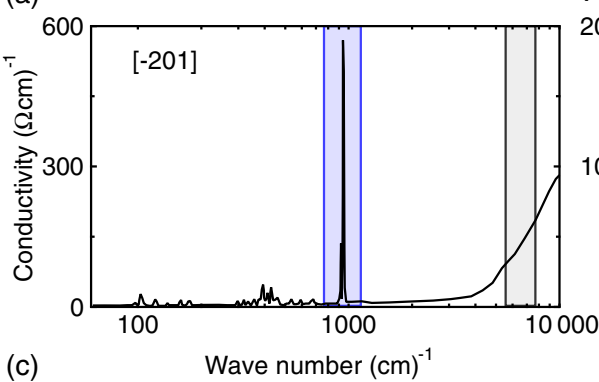

(b)

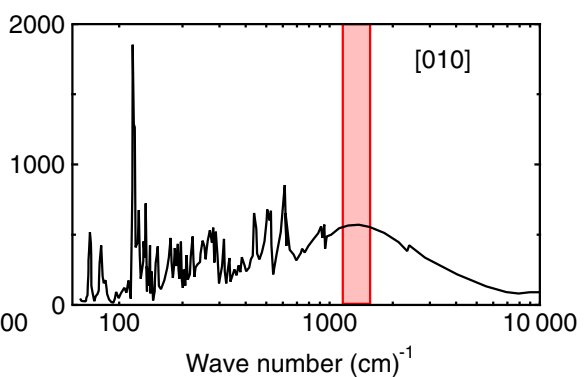

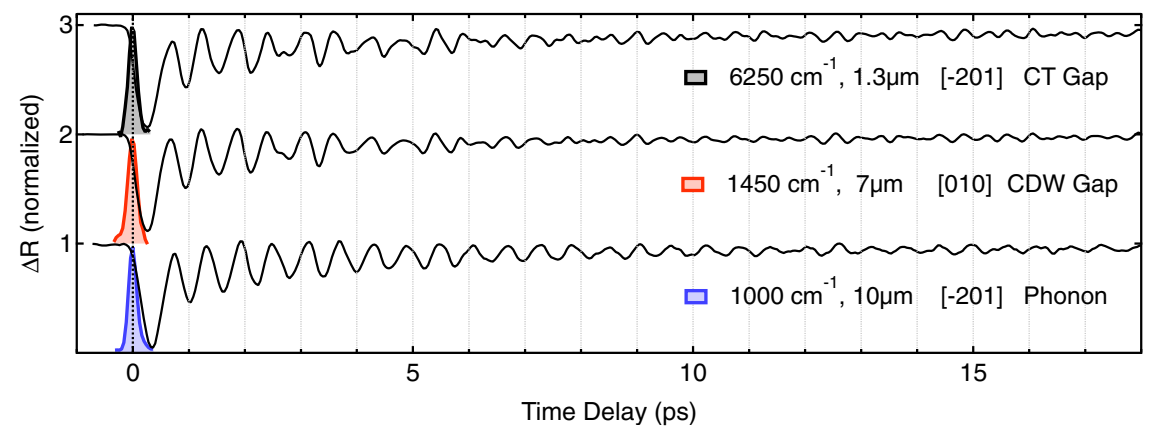

FIG. 2. Optical conductivity $\sigma_{1}$ of $\mathrm{K}_{0.3} \mathrm{MoO}_{3}$ below the transition temperature $T_{C}$. (a) The material is insulating along [-201] with a 1.3-eV charge-transfer gap, and the Mo-O stretching mode appears as a sharp peak at $1000 \mathrm{~cm}^{-1}$. (b) The CDW gap is formed along the [010] chain direction. The shaded regions denote the frequencies to which the optical excitation pulses were tuned: infrared-active phonon mode (blue), electronic excitation across the charge-transfer gap (black), and excitation of quasiparticles above the CDW gap (red). (c) Transient reflectivity changes recorded at $800 \mathrm{~nm}$ probe wavelength (normalized) for the three excitation schemes in a regime of weak perturbation. Zero time delay was determined from the simultaneously measured four-wave mixing signal at frequency $2 \omega_{\text {probe }}+\omega_{\text {pump }}$, which yielded the pump pulse envelopes shown in the figure (shaded). 
(a)

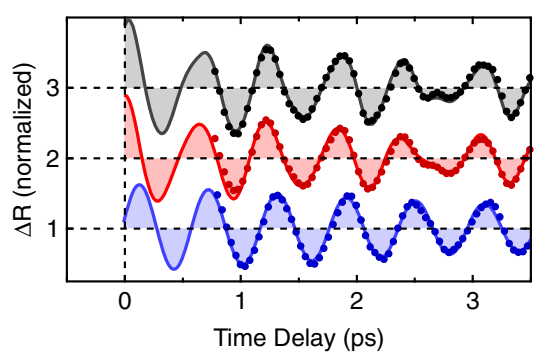

(c)

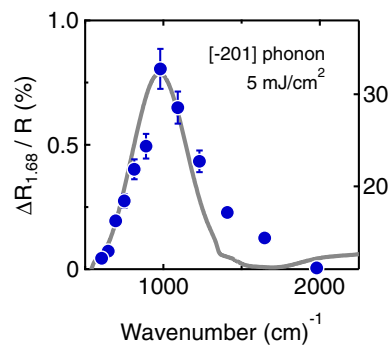

(d) (b)

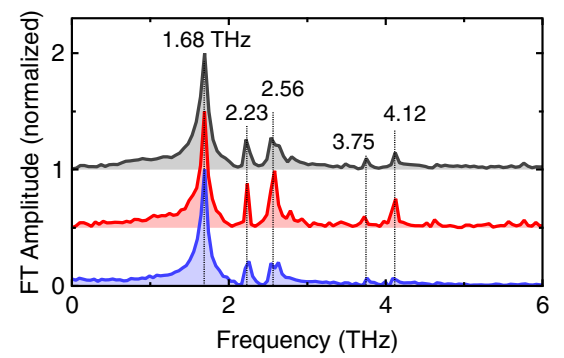

(e)
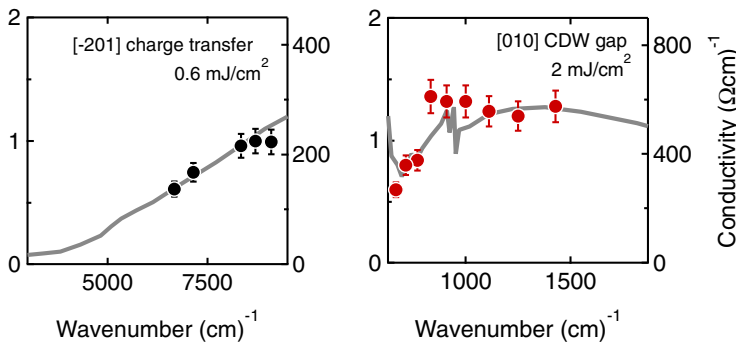

FIG. 3. (a) The normalized oscillatory responses, extracted from the data plotted in Fig. 2(c), are shown as dots. The different optical excitations are color coded as described in Figs. 2(a) and 2(b). The extrapolation of the oscillatory response to time zero, fitted with a sum of three exponentially decaying harmonic oscillators, shows a sinelike phase for lattice excitation and a cosinelike phase for the electronic excitations. (b) Fourier transforms of the oscillatory responses, showing the CDW amplitude mode at $1.68 \mathrm{THz}$ and four zonefolded phonon modes at higher frequencies. (c)-(e) Pump frequency dependent magnitudes of the coherent amplitude mode oscillations, again for the three different excitation schemes. The optical conductivity in the respective frequency ranges and along the respective directions, convolved with the excitation pulse bandwidth is plotted for comparison.

The absolute phase of these oscillations was derived by extrapolating the fit to the pump pulse arrival time at $t=0$. To precisely determine the zero time delay in the different experimental conditions, we simultaneously measured a four-wave mixing signal at frequency $2 \omega_{\text {probe }}+\omega_{\text {pump }}$, which directly yielded the cross-correlation between the pump and probe pulses [see Fig. 3(c)].

The direct excitation of the Mo-O lattice mode resulted in coherent excitation of the amplitude mode with a sine phase, indicative of impulsive excitation, for which the driving force acts on a time scale short compared to the period of the amplitude mode of 600 fs. Assuming lattice anharmonicities as a driving force, such impulsive excitation can be understood if the lifetime of the resonantly excited infrared-active mode is equal to or shorter than the period of the anharmonically coupled amplitude mode [16]. We refer here to previous work on the nonlinear coupling between infrared and Raman modes by nonlinear phononics. In agreement with this picture, the amplitude of the coherent oscillations was found to peak when tuning the frequency of the pump pulses to the resonance frequency of the infrared-active mode as shown in Fig. 3(c) [17].

Cosine phase oscillations were measured for electronic excitations across the [-201] charge transfer (black curve) and the [010] CDW gap (red curve), suggestive of displacive excitation of the coherent amplitude mode $[18,19]$. For these two types of excitation the amplitude of the coherent oscillations closely follows the optical conductivity as function of pump pulse frequency [Figs. 3(d), (e)].

Figure 4 displays the fluence dependence of amplitude, frequency, and scattering rate of the CDW amplitude mode coherent dynamics. All three types of excitation caused the same nonlinear behavior in the optical response [Figs. 4(a), 4(b), 4(c)], showing first a linear increase of the oscillation amplitude, followed by a saturation concomitant with a frequency softening and a nonlinear increase in scattering rate. The threshold value of the excitation energy density, calculated by taking into account reflectivity losses and the penetration depth of the pump pulses, is approximately $3 \mathrm{~J} / \mathrm{cm}^{3}$ for phonon excitation and $30 \mathrm{~J} / \mathrm{cm}^{3}$ for both electronic excitations (see upper panels of Fig. 4). The latter density is similar to values previously obtained in $800 \mathrm{~nm}$ pump experiments (Ref. [6]).

Note also that despite the different threshold values for the three excitation conditions, saturation of the oscillation amplitude was always observed at a change in reflectivity $\Delta R / R_{0} \sim 2 \%$, which was probed by the same $800 \mathrm{~nm}$ wavelength pulses. As the change in optical reflectivity along a coherent phonon coordinate is linearly proportional to the real space atomic displacement, the observation reported here indicates that in all three cases CDW melting occurs for the same displacement of the amplitude mode [19]. This amplitude can be quantitatively estimated by 

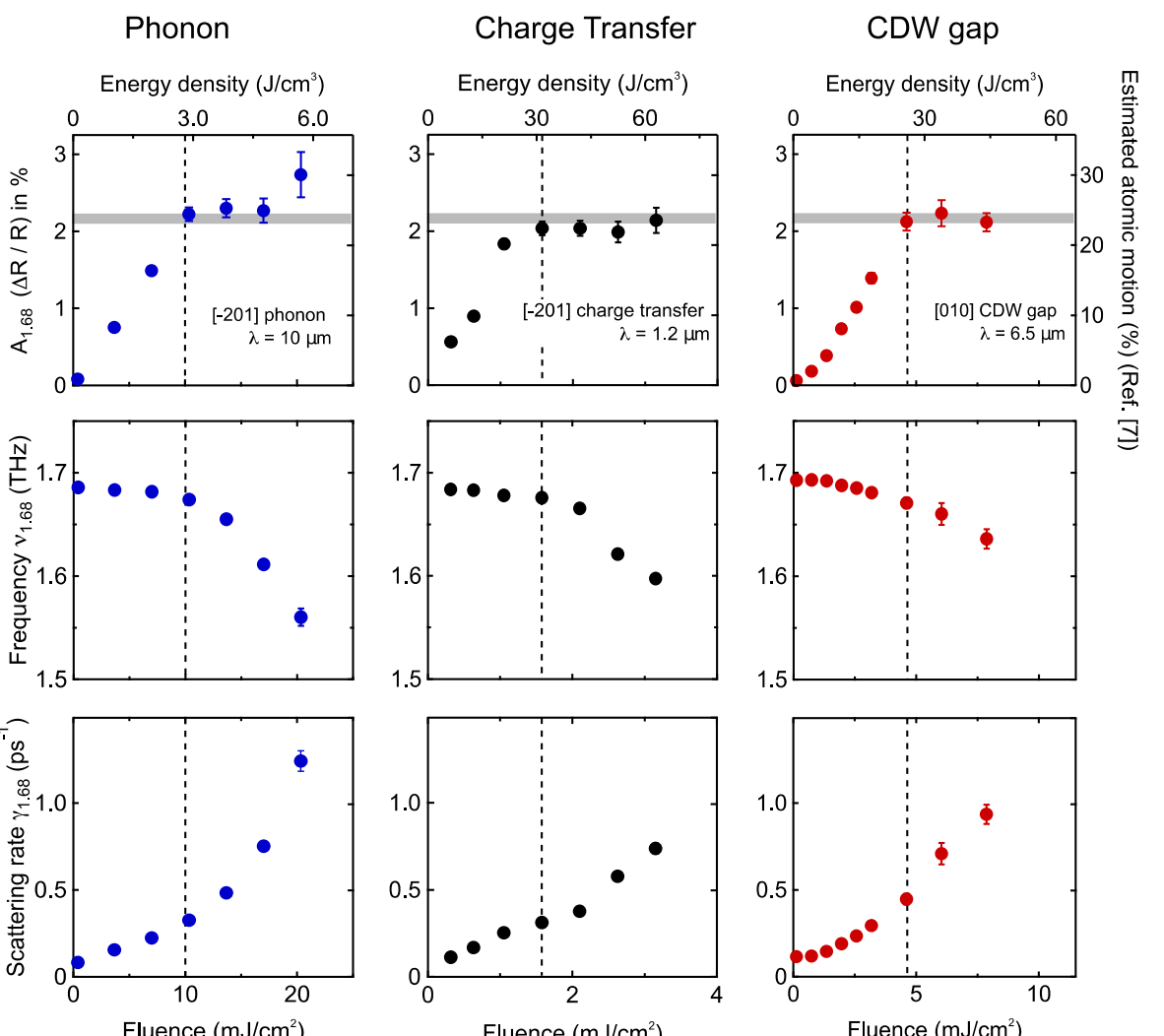

FIG. 4. Fluence dependence of the amplitude, frequency, and scattering rate of the coherent oscillations of the amplitude mode for resonant phonon excitation (blue) and for electronic excitations above the charge transfer gap (black) as well as across the CDW gap (red). The corresponding energy density is given in the top axis of the upper panels. In all cases, the oscillation amplitude saturated at $\sim 2 \% \Delta R / R_{0}$, which corresponds to atomic displacements of approximately $20 \%$ of the lattice distortion induced by the CDW formation at equilibrium as estimated from Refs. [6] and [7] (scale on the right side of upper panel, see text).

comparing previously measured changes in 800-nm optical reflectivity (from Ref. [6]) with femtosecond x-ray diffraction measurements (from Ref. [7]) induced by the same excitation. According to this estimate, the $2 \% \Delta R / R_{0}$ at the CDW melting threshold corresponds to a coherent displacement of the amplitude mode of $22 \%$ of the static lattice distortions that accompany the equilibrium CDW-tometal transition.

The frequency and relaxation was then plotted as a function of the coherent oscillation amplitude. As shown in Fig. 5, both the mode softening and scattering rate fall on a single curve, which indicates abrupt dynamical melting of the charge density wave phase at a critical lattice displacement of approximately $20 \%$ of the equilibrium displacements.

These data provide a new view for the dynamical melting of electronic order, identifying a stability limit for one-dimensional charge density waves. This effect is more reminiscent of the Lindemann criterion for structural melting than of a second order transition, which proceeds by mode softening and divergence of fluctuations. This appears to be also in agreement with studies of charge density wave melting in two dimensional compounds like $\mathrm{TaS}_{2}$, where the collapse of the band gap measured by photoemission [20] and optical spectroscopy [21] appears to be accompanied by melting of the charge density wave [22] at an incomplete relaxation of the lattice [23].
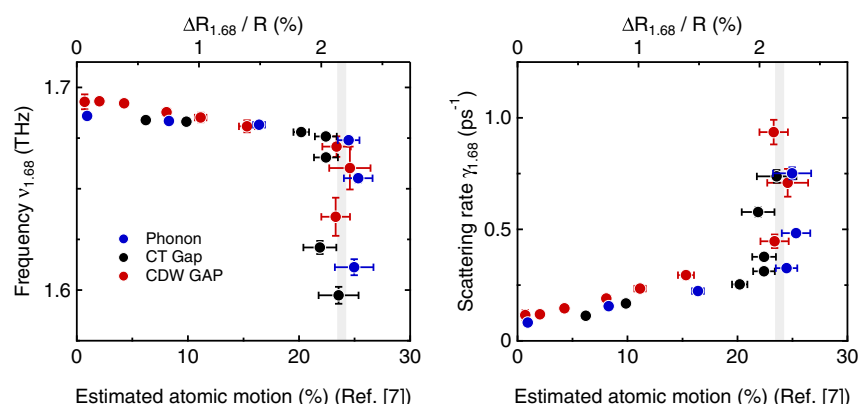

FIG. 5. Frequency and scattering rate of the charge density wave amplitude mode, extracted from the data shown in Fig. 4 and plotted against the measured oscillation amplitude. The data fall on a universal curve for the different excitation schemes. The amplitude of the coherent oscillations is given in $\%\left(\Delta R / R_{0}\right)$ at $800 \mathrm{~nm}$ (upper scale) and in the relative change of the lattice distortion, induced by the CDW formation (lower scale). 
Note also that similar critical displacements could be extracted from previous measurements of the solid-toliquid transition in bismuth [24], which included mode softening [25] above a critical photoexcitation threshold. The present data restrict the observation to a onedimensional system and an additional benchmark for theoretical work, toward a general theory for nonequilibrium phase transitions.

The observations reported here provide also interesting perspective for experiments, in which melting of competing charge density waves with light enhance another order. Indeed, in the case of high-temperature cuprate superconductors, enhancement of superconducting coupling [26-29] was found to follow optical excitation, accompanied by melting of charge order which appears to take place both for excitation at visible frequency [30] as well as with midinfrared pulses [31,32].

The research leading to these results has received funding from the European Research Council under the European Union's Seventh Framework Programme (FP7/ 2007-2013)/ERC Grant Agreement No. 319286 (Q-MAC). This work has been supported by the excellence cluster 'The Hamburg Centre for Ultrafast Imaging - Structure, Dynamics and Control of Matter at the Atomic Scale' of the Deutsche Forschungsgemeinschaft.

[1] R. E. Peierls, Quantum Theory of Solids (Oxford University Press, Oxford, UK, 1955).

[2] G. Grüner, Density Waves in Solids (Addison-Wesley, Reading, MA, 1994).

[3] H. Schaefer, V. V. Kabanov, and J. Demsar, Phys. Rev. B 89, 045106 (2014).

[4] H. Schäfer, V. V. Kabanov, M. Beyer, K. Biljakovic, and J. Demsar, Phys. Rev. Lett. 105, 066402 (2010).

[5] H. Y. Liu, I. Gierz, J. C. Petersen, S. Kaiser, A. Simoncig, A. L. Cavalieri, C. Cacho, I. C. E. Turcu, E. Springate, F. Frassetto, L. Poletto, S. S. Dhesi, Z. A. Xu, T. Cuk, R. Merlin, and A. Cavalleri, Phys. Rev. B 88, 045104 (2013).

[6] A. Tomeljak, H. Schäfer, D. Städter, M. Beyer, K. Biljakovic, and J. Demsar, Phys. Rev. Lett. 102, 066404 (2009).

[7] T. Huber, S. O. Mariager, A. Ferrer, H. Schäfer, J. A. Johnson, S. Grubel, A. Lübcke, L. Huber, T. Kubacka, C. Dornes, C. Laulhe, S. Ravy, G. Ingold, P. Beaud, J. Demsar, and S. L. Johnson, Phys. Rev. Lett. 113, 026401 (2014).

[8] W. J. Schutte and J. L. De Boer, Acta Crystallogr. Sect. B 49, 579 (1993).

[9] M. Sato, M. Fujishita, S. Sato, and S. Hoshino, J. Phys. C 18, 2603 (1985).

[10] J. W. Brill, M. Chung, Y.-K. Kuo, X. Zhan, E. Figueroa, and G. Mozurkewich, Phys. Rev. Lett. 74, 1182 (1995).

[11] G. Travaglini and P. Wachter, Phys. Rev. B 30, 1971 (1984).

[12] G. Travaglini, I. Mörke, and P. Wachter, Solid State Commun. 45, 289 (1983).
[13] D. M. Sagar, D. Fausti, S. Yue, C. A. Kuntscher, S. van Smaalen, and P. H. M. van Loosdrecht, New J. Phys. 10, 023043 (2008).

[14] J. P. Pouget, B. Hennion, C. Escribe-Filippini, and M. Sato, Phys. Rev. B 43, 8421 (1991).

[15] R. Beyer, N. Barisic, and M. Dressel, Physica B (Amsterdam) 407, 1823 (2012).

[16] R. Mankowsky, M. Först, T. Loew, J. Porras, B. Keimer, and A. Cavalleri, Phys. Rev. B 91, 094308 (2015).

[17] M. Först, C. Manzoni, S. Kaiser, Y. Tomioka, Y. Tokura, R. Merlin, and A. Cavalleri, Nat. Phys. 7, 854 (2011).

[18] H. J. Zeiger, J. Vidal, T. K. Cheng, E. P. Ippen, G. Dresselhaus, and M.S. Dresselhaus, Phys. Rev. B 45, 768 (1992).

[19] T. E. Stevens, J. Kuhl, and R. Merlin, Phys. Rev. B 65, 144304 (2002).

[20] L. Perfetti, P. A. Loukakos, M. Lisowski, U. Bovensiepen, H. Berger, S. Biermann, P. S. Cornaglia, A. Georges, and M. Wolf, Phys. Rev. Lett. 97, 067402 (2006).

[21] N. Dean, J. C. Petersen, D. Fausti, R. I. Tobey, S. Kaiser, L. V. Gasparov, H. Berger, and A. Cavalleri, Phys. Rev. Lett. 106, 016401 (2011).

[22] J. C. Petersen, S. Kaiser, N. Dean, A. Simoncig, H. Y. Liu, A. L. Cavalieri, C. Cacho, I. C. E. Turcu, E. Springate, F. Frassetto, L. Poletto, S.S. Dhesi, H. Berger, and A. Cavalleri, Phys. Rev. Lett. 107, 177402 (2011).

[23] M. Eichberger, H. Schäfer, M. Krumova, M. Beyer, J. Demsar, H. Berger, G. Moriena, G. Sciaini, and R. J. D. Miller, Nature (London) 468, 799 (2010).

[24] K. Sokolowski-Tinten, C. Blome, J. Blums, A. Cavalleri, C. Dietrich, A. Tarasevitch, I. Uschmann, E. Förster, M. Kammler, M. Horn-von-Hoegen, and D. von der Linde, Nature (London) 422, 287 (2003).

[25] D. M. Fritz et al., Science 315, 633 (2007).

[26] S. Kaiser, C. R. Hunt, D. Nicoletti, W. Hu, I. Gierz, H. Y. Liu, M. Le Tacon, T. Loew, D. Haug, B. Keimer, and A. Cavalleri, Phys. Rev. B 89, 184516 (2014).

[27] D. Fausti, R. I. Tobey, N. Dean, S. Kaiser, A. Dienst, M. C. Hoffmann, S. Pyon, T. Takayama, H. Takagi, and A. Cavalleri, Science 331, 189 (2011).

[28] D. Nicoletti, E. Casandruc, Y. Laplace, V. Khanna, C. R. Hunt, S. Kaiser, S. S. Dhesi, G. D. Gu, J. P. Hill, and A. Cavalleri, Phys. Rev. B 90, 100503(R) (2014).

[29] E. Casandruc, D. Nicoletti, S. Rajasekaran, Y. Laplace, V. Khanna, G. D. Gu, J. P. Hill, and A. Cavalleri, Phys. Rev. B 91, 174502 (2015).

[30] V. Khanna, R. Mankowsky, M. Petrich, H. Bromberger, S. A. Cavill, E. Möhr-Vorobeva, D. Nicoletti, Y. Laplace, G. D. Gu, J. P. Hill, M. Först, A. Cavalleri, and S. S. Dhesi, Phys. Rev. B 93, 224522 (2016).

[31] M. Först, R. I. Tobey, H. Bromberger, S. B. Wilkins, V. Khanna, A. D. Caviglia, Y.-D. Chuang, W. S. Lee, W. F. Schlotter, J. J. Turner, M. P. Minitti, O. Krupin, Z. J. Xu, J. S. Wen, G. D. Gu, S. S. Dhesi, A. Cavalleri, and J. P. Hill, Phys. Rev. Lett. 112, 157002 (2014).

[32] M. Först, A. Frano, S. Kaiser, R. Mankowsky, C. R. Hunt, J. J. Turner, G. L. Dakovski, M. P. Minitti, J. Robinson, T. Loew, M. Le Tacon, B. Keimer, J. P. Hill, A. Cavalleri, and S. S. Dhesi, Phys. Rev. B 90, 184514 (2014). 